\title{
From paper to paperless: Do electronic systems ensure safe and effective communication and documentation of DNACPR decisions?
}

\author{
Authors: Laura Harrington, ${ }^{A}$ Kathryn Price ${ }^{B}$ and Polly Edmonds ${ }^{C}$
}

\section{Introduction}

An electronic resuscitation system, implemented in 2015, within electronic patient records (EPR) at King's College Hospital NHS Foundation Trust was studied, aiming to review and improve decision documentation and communication.

\section{Method}

The study (January 2018 - June 2018) included all gerontology inpatients with electronic do not attempt cardiopulmonary resuscitation (e-DNACPR) decisions. Cases were identified weekly, followed by retrospective analysis of discharges. Amendments to the electronic system and improvements were implemented between cycles.

Cycle 1

One-hundred and thirty-three patients were included; $85 \%$ had an e-DNACPR form; $86 \%$ of all forms had senior doctor involvement; $68 \%$ evidenced patient/relative discussion; $13 \%$ documented multidisciplinary team (MDT) discussion.

\section{Interventions}

A mandatory 'named nurse' field was added to the form and trust-wide education programme implemented.

\section{Cycle 2}

One-hundred and twenty-six patients were included; $100 \%$ had an e-DNACPR form; 93\% evidenced senior doctor involvement; $\mathbf{7 1 \%}$ evidenced patient/relative discussion; $\mathbf{5 7 \%}$ documented MDT discussion.

\section{Conclusion}

Changes to the process and trust-wide education resulted in more robust documentation and communication.

KEYWORDS: Resuscitation, DNACPR, patient safety, advanced care planning, communication

DOI: 10.7861/clinmed.2019-0450

Authors: ${ }^{\text {A }}$ acute medicine registrar, University College London Hospitals NHS Foundation Trust, London, UK; ${ }^{B}$ care of the elderly registrar, University Hospital Lewisham, London, UK; ' Consultant in palliative medicine, King's College Hospital NHS Foundation Trust, London, UK

\section{Introduction}

NHS England's Five Year Forward View aimed for 'Fully interoperable electronic health records so that patients' records are largely paperless.' by $2020 .^{1,2}$ Complex needs in an ageing population with progressively scarce resources are driving essential technological innovation to support patient safety. With NHS trusts transitioning to paperless, it is vital that integrated electronic systems are safe, efficient, transparent and fit for purpose for our patients.

Do not attempt cardiopulmonary resuscitation (DNACPR) decisions are recommended for patients with progressive illnesses where cardiopulmonary resuscitation (CPR) is unlikely to be successful, to protect them from invasive and potentially inappropriate intervention at the end of life. Inconsistencies in decision making, communication and documentation can lead to misunderstanding between patients, families and healthcare professionals, and can lead to delivery of suboptimal care for some patients. ${ }^{3}$ Greater emphasis on ensuring high-quality and timely communication is recommended in national guidance; therefore, it is vital that accurate electronic documentation is able to support this. 4,5

The 2014 Court of Appeal decision regarding Tracey v Cambridge University Hospitals NHS Foundation Trust changed the requirements for discussing DNACPR decisions with patients and their families. ${ }^{6}$ The Tracey judgment ruled that insufficient communication of this decision breached the patient's human right to knowledge of her treatment. This ruling has been widely publicised in both the media and medical literature, bringing communication to the forefront of healthcare. It is good practice to ensure relatives are aware of DNACPR decisions.

In 2015, King's College Hospital NHS Foundation Trust made the transition to paperless and implemented an electronic system for recording a patient's CPR status. ${ }^{7}$ On accessing the electronic patient record (EPR), a toolbar displays important information, including a patient's CPR status either 'For' or 'DNACPR' (see supplementary material S1). In keeping with national and trust policy, the CPR status should ideally be documented within 24 hours of admission to hospital; if no decision is recorded there is a default that the patient remains 'For' CPR. For a DNACPR decision, the clinician is prompted to complete an electronic DNACPR (e-DNACPR) explanatory form, alongside a treatment escalation plan (TEP). The toolbar is intended for quick reference for all clinical staff. 
In 2016-2017, a number of serious untoward incidents occurred, where patients had inappropriate CPR attempts, despite having valid DNACPR decisions; a subsequent investigation identified a lack of awareness of patients' CPR status on EPR and inconsistent handover of information. This study aimed to review and improve practice in relation to DNACPR documentation and communication on gerontology wards in the trust. ${ }^{8}$

\section{Method}

A single-centred, prospective study was conducted within the three inpatient gerontology wards at an 1,100-bed London teaching hospital. This department was selected because it has a larger number of patients with DNACPR decisions and it was where adverse incidents had previously occurred. Those who were 'For' resuscitation were then excluded from further analysis, as this study aimed to review those with a DNACPR decision only.

Cycle 1 data collection took place in January 2018 and reviewed current practice in relation to the trust's DNACPR policy, which is based on national policy for good medical practice. ${ }^{8}$ Initially, a questionnaire to assess staff familiarity with the policy was distributed to the gerontology team (see Table 1). Questions were designed to identify issues with the e-DNACPR process. Thematic analysis was applied to establish trends in questionnaire responses.

A series of interventions to improve awareness and documentation (see supplementary material S1) took place between January and June 2018, prior to cycle 2 data collection to assess impact. Key interventions included amendment of the e-DNACPR form to add a mandatory 'named nurse' field; amendment of the electronic process such that it could not be finalised without completing the full

Table 1. Staff questionnaire

\section{Question}

Where would you look to find a patient's resuscitation status?

What documentation would suffice for you to NOT perform CPR?

Where would you access the trust policy on DNACPR decisions?

Are you aware of any resources for patients and/or relatives regarding DNACPR decisions? If yes, how would you access them?

What is your understanding of how an inpatient DNACPR decision is translated or communicated to the GP / primary care?

\section{Outcome}

$100 \%$ of respondents answered that they would reference the EPR banner on the 'toolbar' at the top right-hand corner of the patient's record

$58 \%$ EPR toolbar banner alone $31 \%$ DNACPR form $8 \%$ documentation in clinical notes $73 \%$ of respondents knew how to access the trust policy

$27 \%$ of respondents were aware of the current DNACPR / advanced care planning patient leaflet

$77 \%$ of respondents would document in the discharge summary $11 \%$ would print the DNACPR form $11 \%$ would not communicate

$\mathrm{CPR}=$ cardiopulmonary resuscitation; DNACPR $=$ do not attempt cardiopulmonary resuscitation; $E P R=$ electronic patient record; GP = general practitioner.
e-DNACPR form; and implementation of guidance on incorporation of CPR status from EPR at both board rounds and clinical handover. The latter was built into the trust-wide ward improvement and accreditation programme.

In each cycle, data was collected prospectively from EPR weekly over a 4-week period. The e-DNACPR toolbar, explanatory e-DNACPR form, medical notes and discharge letters were reviewed. Documented discussions with patients, families, senior doctors, nursing staff and the wider multidisciplinary team (MDT) were recorded. At week 5 for both cycles, retrospective review of discharge information was undertaken to establish if decisions were communicated to the general practitioner (GP).

Data collection and storage complied with the General Data Protection Regulation. $\chi^{2}$ analysis was used to compare the two cycles statistically. Conclusions from the study were widely presented at national conferences (Society for Acute Medicine and British Geriatric Society) as well as at departmental meetings and grand round (which also acted as vital interventions to educate staff on the topic in further detail).

\section{Results}

The questionnaire response rate was 100\% $(n=26 ; 38 \%$ senior doctors (ST3 registrar or above), $62 \%$ junior doctors (foundation year 1 (FY1) to core medical trainee year 2 (CMT2) doctors)), see Table 1.

Questionnaire response thematic analysis revealed several issues: a need to disseminate the trust policy and resources; training requirements around communication of DNACPR decisions; technical issues with the electronic system; and uncertainty around communication of decisions upon discharge. These factors were considered during the implementation of change and discussion of results.

\section{Documentation of electronic DNACPR decisions}

Cycle 1 included 181 patients and cycle 2 included 176; 100\% of patient notes selected had a resuscitation status ('For' or 'DNACPR') documented within the electronic toolbar.

In cycle 1, 48 (27\%) patients were 'For' resuscitation, 133 (73\%) patients had a DNACPR status. In cycle 2, $50(28 \%)$ patients were 'For' resuscitation, 126 (72\%) patients had a DNACPR status. Therefore, the two samples were comparable. Those 'For' resuscitation were excluded from further data collection, leaving a population sample of 133 patients in cycle 1 and 126 in cycle 2 with DNACPR decisions which were further analysed.

Of the 133 patients in cycle $1,113(85 \%)$ of these had an explanatory e-DNACPR form; 20 (15\%) had no valid e-DNACPR form to support the DNACPR decision. In cycle 2, all (100\%) patients with DNACPR decisions had an explanatory e-DNACPR form. The main findings are shown in Table 2.

\section{When are these decisions made and by whom?}

At the time of the study, the trust policy stated that DNACPR decisions needed to be made by a senior doctor (ST3 registrar or above), with a consultant countersignature. In cycle 1, 115 (86\%) patients had a senior documented decision either on the electronic form or within the medical notes, leaving 20 patients (15\%) with no senior input into a DNACPR decision. Following interventions, $117(93 \%)$ had senior documented input leaving $7 \%$ without. 
Table 2. Summary of results pre- and post-intervention

\begin{tabular}{|c|c|c|c|}
\hline Outcome measure & Cycle 1 (pre-intervention) & Cycle 2 (post-intervention) & $\mathrm{p}$ values \\
\hline Total number of patients, $n$ & 181 & 176 & N/A \\
\hline A CPR status in EPR toolbar, $n(\%)$ & $181(100)$ & $176(100)$ & N/A \\
\hline Number with DNACPR, n (\%) & $133(73)$ & $126(72)$ & $p=0.6$ \\
\hline Explanatory e-DNACPR form present, $\mathrm{n}(\%)$ & $113(85)$ & $126(100)$ & $p<0.0001$ \\
\hline Decision made within 48 hours of admission, $n(\%)$ & $82(62)$ & $91(72)$ & $p=0.07$ \\
\hline DNACPR communicated to GP on discharge, $n(\%)$ & $27(79)$ & $71(90)$ & $p<0.0001$ \\
\hline \multicolumn{4}{|l|}{ Documented discussion with: } \\
\hline patient or relative, $\mathrm{n}(\%)$ & $92(69)$ & $87(69)$ & $p=0.98$ \\
\hline MDT, n (\%) & $17(13)$ & $72(57)$ & $p<0.0001$ \\
\hline senior doctor, ${ }^{a} \mathrm{n}(\%)$ & $115(86)$ & $117(93)$ & $p=0.09$ \\
\hline
\end{tabular}

Over half of DNACPR decisions were made by a 'parent' team within working hours ( $9 \mathrm{am}-5 \mathrm{pm}$, Monday to Friday), ie 78 (59\%) in cycle 1 and $84(67 \%)$ in cycle 2 , with the remaining decisions made by on-call teams ( $41 \%$ in cycle 1 and $33 \%$ in cycle 2 ). In cycle $1,82(62 \%)$ of DNACPR decisions were made within 48 hours of the patient's admission. In cycle 2, 91 (72\%) of decisions were made within 48 hours.

Communication of DNACPR decisions with patients and relatives

In cycle 1, there was evidence of discussion with either the patient or relatives in 91 cases (68\%). There was no documentation of discussion with either in the remaining 42 patients (32\%). For those with no documented patient discussion, 26 (62\%) cited justification for lack of communication eg 'advanced dementia, patient does not have capacity' or 'patient critically unwell with a reduced Glasgow coma scale, no capacity'. While explaining why patient discussions were not possible, there was no justification for lack of discussion with relatives in 16 cases (38\%).

In cycle 2 the number of documented discussions with either the patient or relatives had not significantly improved from cycle 1 (68\% to $69 \%$ ) with a similar number of patients having no documentation of DNACPR discussion (42 (32\%) in cycle 1; 39 patients $(31 \%)$ in cycle 2). Documented reasoning for lack of communication reduced between cycles with only 16 patients $(41 \%)$ having a justification in cycle 2 compared to $26(62 \%)$ in cycle 1 .

\section{Communication of decisions with the MDT}

In cycle 1, $116(13 \%)$ of DNACPR decisions had documented evidence of discussion with the nursing staff. In cycle 2, 72 (57\%) of explanatory e-DNACPR forms referenced a named nurse representing improvement following intervention.

\section{Communication of inpatient DNACPR decisions on discharge to the community}

In cycle 2, a higher proportion of discharged patients had a DNACPR decision (Fig 1). There was an improvement in handover of this decision to the GP in the discharge letter, predominantly utilising the structure of the comprehensive geriatric assessment
(CGA) to prompt documentation of advanced care planning, including DNACPR.

\section{Adverse events}

No adverse events in relation to DNACPR decisions were submitted during the study period; this improvement has been sustained.

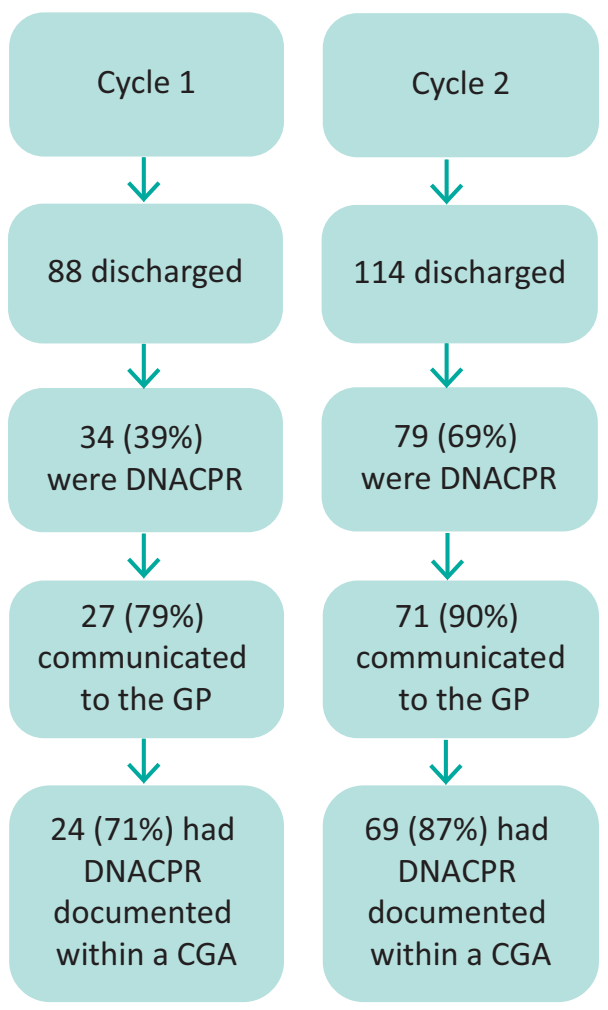

Fig 1. Flow chart of communication of inpatient do not attempt cardiopulmonary resuscitation decisions on discharge to the community. CGA = comprehensive geriatric assessment; DNACPR = do not attempt cardiopulmonary resuscitation; $\mathrm{GP}=$ general practitioner. 


\section{Discussion}

This study demonstrates that changing to electronic documentation of DNACPR decisions can create staff uncertainty, lack of awareness of CPR decisions and suboptimal documentation. Safe and effective implementation of electronic records requires construction of systems that enable highquality record keeping and timely communication. This study demonstrates how introducing electronic documentation is complex, and modification of electronic systems alongside trustwide training and support is required to minimise risks.

Staff questionnaire respondents appeared to be aware of the electronic prompt (DNACPR toolbar) for emergencies; however, staff were unclear whether this was sufficient to guide emergency decision making (Table 1). The survey illustrated a lack of familiarity with the trust DNACPR policy, the supporting resources available and of the electronic systems; this uncertainty has the potential to put patients at risk of inappropriate resuscitation.

Cycle 1 showed that some patients (15\%) did not have an explanatory e-DNACPR form to support the DNACPR decision. This form is required to clearly document the rationale for decisions and subsequent communication. Additional review of the explanatory forms showed insufficient discussion documentation with patients, relatives and the wider MDT, demonstrating the need for improvement overall.

Modest IT changes led to improvements in documentation by ensuring all patients had an explanatory form and by prompting MDT discussion by adding a 'named nurse' section to that form. Improvement of communication with the MDT following intervention was demonstrated; in cycle 2,57\% of decisions had a named nurse documented, despite this mandatory section on the form. It is not possible to control for what is written in these sections, demonstrating a further need for trust-wide training and support.

Despite dissemination of the cycle 1 results in a range of trustwide forums, documentation of discussions with patients and families did not significantly improve and may be partly explained by the uncertainty expressed in the staff questionnaire regarding DNACPR discussions. Therefore, discussion and communication of DNACPR decisions, as part of advanced care planning, remains a substantial challenge.

This study shows that having mandatory electronic items within EPR, such as CPR status, can help encourage decisions to be made on admission (the majority were made within 48 hours); however, this could highlight additional challenges, eg patients being too unwell to contribute to decision, or next of kin being unavailable on admission (particularly out of hours). Encouraging decision making at the 'front door' is trust policy, reflecting the 2012 National Confidential Enquiry into Patient Outcome and Death report Time to intervene? that recommends that CPR status must be considered and recorded for all acute admissions. ${ }^{5}$

An unintended consequence of early CPR decision making, as seen in this study, is that CPR decisions may be made without full discussion with patients and their families, which could lead to misinterpretation or distress. This highlights a further need to educate and support staff when implementing new electronic systems; more training is needed to enable high-quality discussions and ensure good communication of decisions in a high-pressure environment. More work is needed nationally to raise awareness of dying as a natural process and in understanding the limitations of resuscitation in patients with poor physiological reserve and life-limiting illness. It is critical to empower patients, to allow informed decision making and ensure patients/families feel comfortable expressing their wishes.

Following this study, our trust policy was amended to recommend that CPR/DNACPR decisions are reviewed when patients transfer to new clinical areas, allowing doctors to review appropriateness of decisions and the extent of communication with patients. Arrival in a new clinical setting provides opportunity for review of care goals, CPR decisions and patient preferences. In busy clinical settings, user-friendly and simple DNACPR documentation is necessary to ensure efficiency.

The $44 \%$ improvement in MDT discussion is encouraging. Specific guidance was developed to support safe handover on transfer and shift changes, aiming to minimise the chances of miscommunication and inappropriate resuscitation in the event of clinical deterioration. The gerontology wards had enthusiastic teams, which is likely to have underpinned the project's success. Wider key stakeholder engagement increased the effectiveness of changes instigated and facilitated MDT and senior matron-led focus group impact to incite trust-wide cultural change.

The use of CGAs (and subsequently advanced care planning decisions) within discharge letters encouraged communication to GPs, although it is essential that decisions are discussed with patients and families prior to inclusion in discharge letters to prevent distress if not previously known or if misunderstood. More work is needed to improve communication with primary care, the ambulance service and social care to effectively communicate advanced care decisions appropriately.

\section{Limitations and future work}

The study's findings may not be generalisable as they relate to the electronic documentation of CPR decisions in one clinical area within an acute trust. However, as trusts become increasingly paperless, these findings could highlight potential issues to overcome when moving from paper-based to electronic systems, in this case for DNACPR documentation. Our experience demonstrated that while robust systems are needed, the cultural change and staff training required to support the move to electronic systems cannot be underestimated.

The information gained from electronic systems is limited by the quality of input; in this study we were not able to review the quality of the communication interface or the appropriateness of clinical decision making. We excluded those 'For' CPR in this study and therefore cannot apply conclusions to these patients. Future work could be undertaken to assess the challenges in making timely CPR decisions, ensuring clear communication, recognising patient wishes and explaining decisions to optimise communication with patients and their families.

\section{Conclusion}

This study was prompted by a series of adverse events following the implementation of electronic CPR documentation and demonstrated that significant changes in practice when moving to electronic systems can create uncertainty among staff. Improvements in the EPR process and development of robust handover systems led to sustainable and effective change.

However, improving the quality of clinical decision making around DNACPR remains challenging given its inherent sensitivity, therefore national education on the benefits of resuscitation in people with life-limiting illness and limited reversibility still requires work. 
Locally, improved documentation could be encouraged through pop-up prompts for blank fields on e-DNACPR forms, further supporting the review and re-discussion of CPR status on transfer to new clinical environments. However, evaluation of such improvements is limited by the quality of documentation inputs.

This audit tool has application to other hospital areas and lessons learnt can be applied to other trusts implementing e-DNACPRs. Future work is needed to evaluate communication quality and encourage communication of DNACPR decisions to the community via CGAs in discharge summaries, with ongoing improvement in electronic systems to reflect these needs. This can then be translated into a wider understanding of DNACPR decisions and advanced care planning for patients both in the community and in hospital.

\section{Supplementary material}

Additional supplementary material may be found in the online version of this article at www.rcpjournals.org/clinmedicine:

S1 - An example of an electronic patient record and summary of interventions.

\section{References}

1 NHS England. Five Year Forward View. NHS, 2014.

2 NHS, National information Board, The forward view into action: Paper-free at the point of care, guidance for developing local digital roadmaps. NHS, 2016. www.england.nhs.uk/digitaltechnologyold/wp-content/uploads/sites/31/2016/11/develp-ldrs-guid.pdf
3 Fritz Z, Slowther AM, Perkins G. Resuscitation policy should focus on the patient, not the decision. BMJ 2017;356:j813.

4 British Medical Association, Resuscitation Council (UK), Royal College of Nursing. Decisions relating to cardiopulmonary resuscitation. London: Resuscitation Council (UK), 2016. www.resus.org.uk/ dnacpr/decisions-relating-to-cpr

5 National Confidential Enquiry into Patient Outcome and Death. Time to intervene? A review of patients who underwent cardiopulmonary resuscitation as a result of an in-hospital cardiac arrest. NCEPOD, 2012. www.ncepod.org.uk/2012report1/downloads/ CAP_fullreport.pdf

6 Szawarski P. Classic cases revisited: Mrs Janet Tracey, Resuscitation and the importance of good communication. J Intensive Care Soc 2015;16:142-6.

7 Johnson M, Whyte M, Loveridge R, Yorke R, Naleem S. A unified electronic tool for CPR and emergency treatment escalation plans improves communication and early collaborative decision making for acute hospital admissions. BMJ Qual Improv Rep 2017:6:u213254.w6626.

8 Edmonds P, Burman R, End of Life Strategy Care Group. Do not attempt cardiopulmonary resuscitation policy. London: King's College Hospital Hospital NHS Foundation Trust, 2017.

Address for correspondence: Dr Laura Harrington, Acute Medical Unit, University College Hospital, 235 Euston Road, Bloomsbury, London NW1 2BU, UK.

Email: laura.harrington6@nhs.net

\section{Royal College of Physicians

\section{Acute care toolkit 15: Managing acute care problems in pregnancy}

\section{Free} toolkit

Pregnant women can present to any acute hospital service at any time during their pregnancy or the postpartum period, up to 12 months post-delivery. Women may present with acute medical problems that need to be managed differently because of pregnancy, or may present with obstetric syndromes.

This toolkit provides practical guidance on managing women with acute medical problems in pregnancy. It also advises healthcare staff to take the opportunity to ask about a woman's mental wellbeing when she comes to hospital for other reasons during or after pregnancy; women with a history of mental health problems are more likely to develop new symptoms during pregnancy and postpartum.

Essential reading for front-line NHS staff who may be unfamiliar with the normal physiology of pregnancy and/or diseases that present in pregnancy.

\section{Download now at: www.rcplondon.ac.uk/act15}
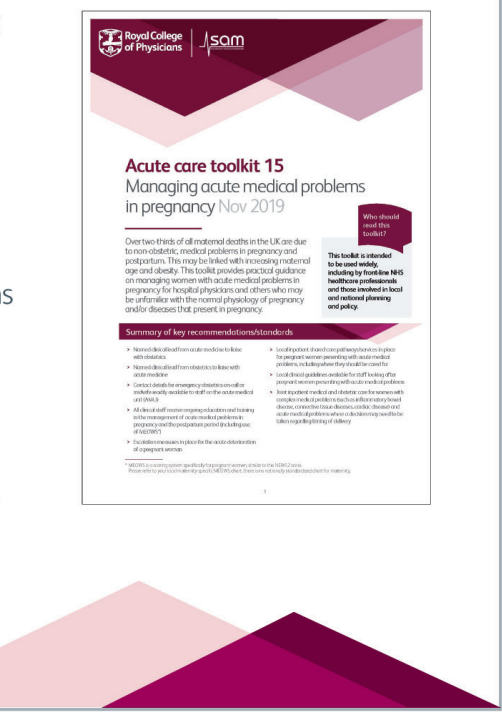